\title{
Philosophiques
}

\section{J.-Fr. Kervégan et B. Mabille (dir.), Hegel au présent : une relève de la métaphysique? CNRS Éditions, Paris, 2012, 460 p.}

\section{Jeanne Allard}

Volume 40, numéro 1, printemps 2013

URI : https://id.erudit.org/iderudit/1018385ar

DOI : https://doi.org/10.7202/1018385ar

Aller au sommaire du numéro

Éditeur(s)

Société de philosophie du Québec

ISSN

0316-2923 (imprimé)

1492-1391 (numérique)

Découvrir la revue

Citer ce compte rendu

Allard, J. (2013). Compte rendu de [J.-Fr. Kervégan et B. Mabille (dir.), Hegel au présent : une relève de la métaphysique ? CNRS Éditions, Paris, 2012, 460 p.]

Philosophiques, 40(1), 229-234. https://doi.org/10.7202/1018385ar

Ce document est protégé par la loi sur le droit d'auteur. L'utilisation des services d'Érudit (y compris la reproduction) est assujettie à sa politique d'utilisation que vous pouvez consulter en ligne.

https://apropos.erudit.org/fr/usagers/politique-dutilisation/ 


\title{
Comptes rendus
}

\author{
J.-Fr. Kervégan et $\mathrm{B}$. Mabille (dir.), Hegel au présent: une relève de \\ la métaphysique? CNRS Éditions, Paris, 2012, $460 \mathrm{p}$.
}

Constitué des actes d'un colloque tenu à Paris et à Poitiers en juin 2009, Hegel au présent s'étend sur 460 pages et comporte 24 collaborations dont la longueur varie de ro à 34 pages, partagées en deux sections: l'une portant sur des analyses internes du propos hégélien, l'autre sur des approches comparatives. Le titre rappelle celui d'une contribution antérieure au corpus des études hégéliennes, à laquelle avaient participé Jean-François Kervégan et Emmanuel Renault, parue en I995 sous la direction de H. Maler, intitulée Hegel passé, Hegel à venir. L'enjeu de ce précédent recueil — «faire travailler le texte de Hegel sur les chantiers actuels de la pensée ${ }^{1} »-$ trouve un écho dans le présent ouvrage, qui spécifie davantage la question posée: à tenter d'actualiser la pensée hégélienne, ne la priverait-on pas de sa substance, c'est-à-dire de son caractère métaphysique? Le caractère hégélien de cette reprise, dans laquelle est incorporée une différence, semble marqué par le soustitre où le substantif « relève» évoque la traduction derridienne de Aufhebung. La différence ci-incorporée peut être identifiée à l'importance qu'a acquis pour la philosophie hégélienne, depuis 1995 , le courant pragmatiste et néopragmatiste. Le numéro 99 de la revue Philosophie paru en 2008, Hegel pragmatiste? où étaient traduits des textes d'auteurs anglo-saxons, confirma et nourrit la présence de l'interprétation pragmatiste et néo-pragmatiste de certaines thèses hégéliennes hors du contexte analytique dont elles ont émergé. Le volume s'inscrit donc dans une problématique déjà commentée ponctuellement, mais reste le premier du genre en langue française.

La présentation écrite en commun par Jean-François Kervégan et Bernard Mabile combine leurs deux positions et porte essentiellement sur le traitement de l'hégélianisme nécessaire au renouvellement des recherches sur le lien entre philosophie hégélienne et métaphysique. Ce traitement consiste à présenter l'histoire des études hégéliennes comme la division, non entre jeunes et vieux hégéliens, mais entre l'identification du système spéculatif à la métaphysique et, au contraire, sa rénovation hors d'elle. Au-delà de l'opposition entre conservation et transformation, les interprétations contemporaines sont ainsi plutôt à répartir entre une reprise dynamique de la philosophie hégélienne d'inspiration pragmatiste et une interprétation soucieuse de préserver le caractère théologique et ontologique de la lettre du

1. H. Maler, «Hegel passé, Hegel à venir » dans Hegel passé, Hegel à venir, L’Harmattan, Paris, 1995, p. 9. 
texte hégélienne - bien que les auteurs manifestent (à raison) de l'hésitation à ranger dans cette dernière catégorie les travaux de Bernard Bourgeois, Hans Friedrich Fulda et Dieter Henrich. En effet, le souci interprétatif de rendre le caractère systématique de la philosophie hégélienne n'est pas nécessairement une défense de ce dernier.

L'unité partagée par les textes est thématique, les articles n'ayant d'autre point commun que de répondre à la même question. Ainsi, rarement a-t-on pu observer une telle diversité de traitements et de méthodologies. À titre d'exemple, les textes d'Emmanuel Cattin et de Pirmin Stekeler-Weithofer diffèrent en leur démarche comme en leur forme, et leur lecture contiguë crée l'impression d'une fascinante divergence d'interprétation. Cette diversité donne une idée de l'étendue, de la fertilité et de l'actualité philosophique des études hégéliennes. Cela dit, elle fait également en sorte que je ne pourrai traiter que de quelques-unes des contributions présentées, même si elles sont toutes importantes. Afin de donner l'éventail le plus large, je procéderai en quatre temps en traitant de l'analyse d'un point de vue méthodologique, du lien entre critique et dialectique, de l'interprétation néo-pragmatiste, et enfin de la question de la philosophie première chez Hegel.

En premier lieu, eu égard à la méthode, le texte de Fulda figurant dans le volume constitue une analyse où la prise de position se limite chirurgicalement à la précision du sens de la relation entre la métaphysique et la philosophie hégélienne. L'exploration qu’il propose ( «La philosophie de Hegel — avec la métaphysique et sans elle») repose sur l'impossibilité de choisir entre l'une ou l'autre de ces possibilités, fidèle à la critique hégélienne de la philosophie du «ou bien... ou bien... » selon laquelle cette alternative n'est que l'énoncé d'une contingence et non celui d'une nécessité. Le rapport double à la métaphysique est structuré par l'écho de l'exigence aristotélicienne pour laquelle la métaphysique, en se définissant, s'accompagne d'une discipline et de règles propres qui y correspondent. Cette exigence rappelle l'importance de comprendre la philosophie hégélienne dans ses aspects projectifs et programmatiques. Car les disciplines métaphysiques (Fulda cite la psychologie et la cosmologie rationnelles) ne proviennent pas génétiquement de la logique spéculative, mais lui sont liées comme modes de connaissance uniquement. Il est alors impossible de pratiquer la philosophie sans métaphysique, mais impossible de le faire avec la métaphysique sans se livrer à une typologie des modes de connaissance.

Ce souci de distinction typologique pose, sur un autre plan, la question de l'unité. N'est-ce pas au nom de l'unité que Hegel refuse la possibilité de toute alternative disjonctive en métaphysique? L'analyse d'Annette Sell («Les déficiences de l'ancienne métaphysique: la critique hégélienne de la métaphysique dans le "Concept préliminaire" à la logique de l'Encyclopédie») s'appuie sur une interprétation de la critique que Hegel adresse, dans le Concept préliminaire, aux trois formes de la position de la pensée par rapport à l'objectivité. Cette critique porterait, en ce qui concerne la métaphy- 
sique, sur l'unilatéralité du rapport qui mène la pensée à ne prendre que la finité pour objet. Cette interprétation de la critique de la finité demande toutefois à être justifiée puisque la pensée spéculative, qui tirera son essence de ces critiques, n'est pas seulement ou prioritairement traitement de l'infinité, mais aussi processus de pensée plutôt que prédication, unité de la pensée et de l'objet pensé plutôt que description de leur différence, et devenir de l'être et du néant plutôt que leur opposition. Par cela, le texte de Sell illustre la difficulté devant laquelle est placé le lecteur qui tente de déterminer les rapports entre critique et défense de la métaphysique chez Hegel. Les rapports négatif et positif au même objet doivent être différenciés, sans pour autant que cette différenciation mette à mal l'impératif d'unité spéculative. L'analyse de Fulda dissolvait le problème en prenant appui sur un postulat méthodologique où la disjonction était rejetée; cependant cela ramenait la philosophie avec métaphysique à un principe de division.

En second lieu, il est relativement rare au sein des études hégéliennes qu'on traite le thème de la critique sans qu'il soit mis en relation avec le projet du criticisme kantien, lui-même rejeté par Hegel. Le texte de Renault ( «Deux critiques et deux sauvetages de la métaphysique») propose une analyse intéressante de la différence entre la critique et la défense de la métaphysique. Bien que la première partie de l'analyse portant sur la classification des critiques exposées dans le Concept préliminaire n'apporte pas d'élément nouveau, la seconde partie où est introduite la notion de réconciliation me paraît d'un vif intérêt ${ }^{2}$. Elle rappelle en effet que l'effectivité est spéculative et logique tout autant qu'éthique: si le devoir-être est présent dans les Principes de la philosophie du droit, il l'est aussi dans la Doctrine de l'être, où il a pour fonction de caractériser la limite de l'être déterminés. Chez Renault, cette réconciliation entre esprit théorique et esprit pratique ne survit pas à la critique adornienne, mais demeure comme utopie d'une réalité plus véritable au nom de laquelle il est possible de critiquer. La discussion de cette possibilité de réconciliation m'a toutefois paru courte, bien qu'originale, quant au traitement du sens métaphysique de la philosophie hégélienne.

2. Dans la préface de L'effectif et le rationnel, Jean-François Kervégan fonde justement son interprétation non métaphysique sur le fait que l'optimisme hégélien, c'est-à-dire la conviction que la possibilité d'une réconciliation objective est contredite par l'état du monde. Renault montre, à l'inverse, que, critiquée, elle peut être sauvegardée.

3. Devant l'ampleur de l'ouvrage, j'ai dû privilégier les contributions dont le propos me semblait le plus près de la recherche d'unité entre la critique de la métaphysique et sa défense. Cela a occasionné plusieurs choix déchirants, comme dans le cas du texte de Felix Duque où la logique hégélienne est abordée à partir du concept de liberté. En montrant que la liberté repose toujours sur la vérité, Duque expose les sens multiples de la libération dont il est question: cette libération est une qualité subjective, au sens où le sujet est libre, mais est également libération de quelque chose (en l'occurrence, du monde sensible), et libération par laquelle l'humanisation du monde est produite. 
Dans «Hegel sans la dialectique? », Foessel donne un tout autre sens à l'enjeu séparant la conservation de la transformation de la métaphysique. Ici, la métaphysique est rapprochée de la dialectique (cette analyse, bien que discutable, porte ses fruits), en sorte que le texte s'ouvre sur la possibilité de concevoir la philosophie hégélienne sans dialectique. La dialectique est à la fois le mouvement qui permet la critique de la métaphysique, et le nœud d'une multiplicité de sens que le système hégélien peine à ordonner entre eux. Le véritable sens critique de la dialectique, soutient Foessel, repose non sur le fait de porter sur un élément qui lui est extérieur, mais sur sa capacité à démontrer la contradiction interne d'un élément qui, en cela, montre sa limite. Ce sens de la critique a l'avantage de se rapprocher du rôle attribué à la philosophie dans la préface des Principes de la philosophie du droit ${ }^{4}$ et offre ainsi une assise solide pour poser à la philosophie hégélienne la question de la métaphysique, c'est-à-dire tenter de savoir en lesquels de ses sens la métaphysique est contradictoire avec la philosophie hégélienne, et quelle est la limite tracée par cette nouvelle positivité spéculative.

À ce sujet, en troisième lieu, l'article de Terry Pinkard ( «La liberté et le monde de la vie») porte sur la notion de vie, plus spécifiquement sur la notion d'action libre, qui offre un pont judicieux entre pragmatisme et idéalisme; il est séparé en trois sections de trois parties chacune, mimant l'avancée dialectique en triade. La lecture est d'une grande finesse et effectue plusieurs mises au point (par exemple, celle sur la téléologie historique de l'esprit qui, bien qu'il tende à une fin comme en témoigne son progrès, ne le fait pas consciemment ou subjectivement). De plus, l'insistance de l'auteur à ramener les problèmes hégéliens à des explications proprement linguistiques est, je crois, d'une grande pédagogie. Il demeure que l'approche de la philosophie hégélienne par l'action, la liberté et la quotidienneté concorde facilement avec les Principes de la philosophie du droit (cette concordance est aussi possible du point de vue de la Phénoménologie de l'esprit), mais la transposition des thèses qu'elle permet de développer sur la logique hégélienne, notamment en ce qui touche l'esprit, me semble moins aisée. Par exemple, l'argument hégélien de l'identité spéculative de l'intérieur et de l'extérieur devient, en termes pragmatistes, non l'identité processuelle atteinte par la dialectique, mais la relation entre un agent (intérieur) qui se dédouble dans son action (extérieure). Il est vrai qu'on peut voir dans cela, selon la perspective, le jeu de l'interprétation ou une judicieuse «simplification ${ }^{5}$ ». Je crois néanmoins qu'il demeure possible de critiquer la thèse de Pinkard selon

4. La philosophie ne doit pas poser, de l'extérieur, une théorie sur l'état; car il est faux de poser la réflexion éthique comme recherche d'une vérité «en dehors» du monde éthique. $C f$. Principes de la philosophie du droit (trad. de J.-Fr. Kervégan), G.W. F. Hegel, PUF, Paris, 2003, p. 94-96.

5. T. Pinkard, «Le pragmatisme fut-il le successeur de l'idéalisme?» dans Philosophie, 99, septembre 2008 , p. 36 . 
laquelle le lien entre effectif et rationnel a pour but, chez Hegel, de cautionner la priorité interprétative de l'agentivité (limitée) sur la pensée (totale), alors que l'effectivité se borne à affirmer leur unité.

En tout dernier lieu, il serait impensable de conclure ce texte sans dire un mot de la contribution de Bernard Mabille ( «Hegel, le dépassement de quelle métaphysique? »), qui mène depuis plusieurs années des recherches à partir de la notion de philosophie première. Il expose ici une interprétation aporématique de la philosophie hégélienne, laquelle consiste à suspendre son jugement au sujet de l'évaluation hégélienne de la métaphysique et à interpréter le système hégélien comme un "accueil de déterminations ${ }^{6}$ ". Cette entreprise philosophique méritant d'être présentée plus amplement ${ }^{7}$ que l'espace alloué ici ne le permet, je me bornerai à dire qu'il s'agit d'un des seuls textes (avec celui d'Emmanuel Cattin, bien qu'en un sens différent) où la méthode se fond dans la recherche philosophique proprement dite. Cette transfiguration de la position méthodologique qui refuse d'être indifférente à son objet et qui se caractérise par la recherche d'un sol poursuit avec sens et rigueur le travail qui consiste à comprendre la philosophie hégélienne dans son rapport avec les philosophies de l'Antiquité. Cette compréhension s'est faite discrète dans le monde francophone dans les vingt dernières années (c'est-à-dire depuis la parution de la thèse d'André Doz, environ); ce faisant, on a plus ou moins cessé de dire que le lien avec la philosophie grecque était de première importance pour Hegel lui-même.

Enfin, un mot pour mentionner, en vrac, quelques éléments du reste: l'article d'Olivier Tinland donne un avant-goût intéressant de son prochain ouvrage (dont la sortie est prévue fin 2012 , donc imminente); le texte de Pirmin Stekeler-Weithofer, dans une interprétation purement analytique de la pensée hégélienne, est singulier et mériterait une bien plus vaste analyse; enfin, les articles de Haas et de Lardic offrent une contrepartie originale aux analyses récentes de la Science de la logique.

On peut regretter le manque de références récentes sur la question de la métaphysique chez Hegel (puisque beaucoup d'ouvrages ont porté sur ce sujet, particulièrement en langue anglaise et en langue allemande); de même, un index aurait été opportun, même si cette pratique éditoriale est quasiinexistante lorsqu'il est question d'actes de colloque. Il faut aussi souligner que, bien qu'Axel Honneth ait participé au colloque de 2009 (et que le texte de Michaël Foessel s'en prenne de front à son interprétation des Principes de la philosophie du droit dans Leiden an Umbestimmtheit), son texte ne figure

6. B. Mabille, «Hegel, le dépassement de quelle métaphysique?» dans Hegel au présent: une relève de la métaphysique? J.-Fr. Kervégan et $\mathrm{B}$. Mabille (dir.), CNRS Éditions, Paris, 20I 2 , p. $33 \mathrm{I}$.

7. La suspension du jugement connotant la reprise sceptique employée par Hegel comme repoussoir pour définir la nécessité du commencement, il conviendrait, par exemple, d'évaluer cette piste interprétative à la lumière de la critique hégélienne de l'alternative «ou bien..., ou bien...". 
pas dans le volume. Sur le fond, si la question du pragmatisme est centrale aux yeux des interprètes non métaphysiques, on peut se demander si les arguments du processualisme de Whitehead ne pourraient pas contribuer à ces recherches, de même que le rapprochement tout récent entre la philosophie hégélienne et les modèles contemporains en physique théorique. La première n'est mentionnée qu'une fois (dans la note i9 de l'article de Renault) et la seconde ne l'est pas du tout. Les critiques du lien entre pensée hégélienne et pragmatisme sont nombreuses en langue anglaise, mais peu apparentes ici $^{8}$. Le problème méthodologique qui peut, au début, créer un effet de circularité (pour situer Hegel par rapport à la métaphysique, il faut définir celle-ci; pour définir la métaphysique, il faut situer la pensée hégélienne par rapport à elle), se dissipe rapidement devant le très vaste éventail de réponses proposées. On ne peut reprocher au volume de manquer d'une unité à laquelle il ne prétend pas et qu'il se défend bien de rechercher. Le tout est impressionnant et témoigne d'une pensée vive. On peut très justement le comparer en importance au collectif dirigé par Alasdair MacIntyre, Hegel: A Collection of Critical Essays, en I97I.

JEANNE ALLARD

Université de Montréal

\section{Ludwig Feuerbach, Éthique: l'Eudémonisme, 1867-1869 suivi de L'homme est ce qu'il mange, 1862. Traduction d'Anne-Marie Pin, préface de Roger Bruyeron, Paris, Hermann, 2012, 140 p.}

Essentiellement connu par le lectorat francophone pour son œuvre maîtresse L'essence du christianisme (I84I), Ludwig Feuerbach (I804-I872) eut une influence certaine, bien que trop souvent ignorée, sur tout un pan de la philosophie post-hégélienne. Louis Althusser écrivait en effet: "On constatera avec quelle étonnante profondeur Feuerbach annonce et devance, à un titre ou à un autre, par contraste ou parenté, Marx, Nietzsche, Freud, Husserl, certains thèmes de Heidegger, Barth et la théologie récente ${ }^{9}$.» Cette perspec-

8. Entre autres, Houlgate, S. "Phenomenology and de re interpretation: a critique of Brandom's reading of Hegel»; Stern, R. "Hegel and Pragmatism» dans A Companion to Hegel, Wiley-Blackwell, Oxford, 20I I, p. 556-575 et Hegelian Metaphysics, Oxford University Press, Oxford, 20I 2, spécialement p. 48-50; Redding, P. «The Analytic Neo-Hegelianism of John McDowell and Robert Brandom» dans A Companion to Hegel, Wiley-Blackwell, Oxford, 20I I, p. 576-593 et "Analytic Philosophy and the Return of Hegelian Thought", Cambridge University Press, Cambridge, 2010 [2007]; Macdonald, I. "Nature and Spirit in Hegel's Anthropology: Some Idealist Themes in Hegel's Pragmatism» dans Laval théologique et philosophique, 63: I, février 2007, p. 4I-50. Il faut souligner, à la défense des auteurs, que ces critiques sont, pour la plupart, antérieures à la parution du présent volume, mais postérieures à la tenue du colloque de 2009 .

9. Cité dans la préface de Roger Bruyeron, p. 32. 